\title{
The Energy-Momentum Tensor near a Black Hole`
}

\author{
M. S. Fawcett \\ Department of Mathematics, University of Otago, Dunedin, New Zealand
}

\begin{abstract}
The energy-momentum tensor for a conformally invariant scalar field near a Schwarzschild black hole in thermal equilibrium with radiation is found by a combination of analytical and numerical techniques. Calculations are performed in the Euclidean section of the spacetime, and divergences isolated using the heat kernel expansion. It is found that the results agree well with those of Candelas [1], but that there are significant differences from the Gaussian approximation of Page [2].
\end{abstract}

\section{Introduction}

This paper describes the calculation of the expectation value of the energymomentum tensor, to one loop, for a conformally coupled scalar field near a Schwarzschild black hole in the Hartle-Hawking "vacuum" state - i.e. for a black hole in (unstable) equilibrium with a bath of thermal radiation. Much preliminary work has been done on this problem, and there is a large literature on black hole radiance and the quantum properties of black hole spacetimes, and on quantum field theory in more general curved spacetimes. The original work on black hole radiance was done by Hawking [3] and Hartle and Hawking [4]. Boulware [5], Unruh [6], and Wald [7] further discuss black hole emission and different possible vacuum states. Davies et al. [8], Christensen and Fulling [9], and Candelas [1] discuss the energy-momentum tensor of a scalar field near a black hole. Gibbons and Perry [10] discuss the thermal properties of black holes, and thermal Green functions. There are of course many other papers, references to which can be found in the reviews, e.g. by Gibbons $[11,12]$, De Witt $[13,14]$, and the recent book by Birrell and Davies [15]. These reviews also discuss methods of regularising the expressions for physical quantities in a general spacetime.

The calculation of $\left\langle T_{\mu \nu}\right\rangle$ could have been done years ago - the delay has probably been due to the (perhaps very reasonable) reluctance of people to get

\footnotetext{
* This paper was written while the author was at the DAMTP, Cambridge University, Silver Street, Cambridge, England
} 
involved in numerical calculations. The wave equation in Schwarzschild spacetime is separable but not soluble exactly - one ordinary differential equation requires numerical solution. Once numerical work is required, one must recast the whole problem into a form suitable for numerical evaluation. Thus the methods I use are slightly different from those that generally occur in discussions of black hole quantum theory.

The calculation is done assuming a fixed static background metric, in the presence of which fluctuations of the scalar field are quantised. The back-reaction of the scalar field on the geometry is ignored. Discussions of the usefulness and consistency of doing quantum field theory in a curved spacetime can be found in e.g. Gibbons [12] or Birrell and Davies [15]. My calculations refer to a black hole in thermal equilibrium with radiation. To obtain the results for a black hole radiating into empty space (i.e. in the Unruh vacuum) it would be necessary to subtract the contribution due to incoming thermal radiation. This calculation has not yet been done. This will yield an energy-momentum tensor that can be used to discuss the evolution of the horizon of a black hole as it evaporates, by using it as a source in Einstein's equations. The assumption of a static background field in calculating $\left\langle T_{\mu \nu}\right\rangle$ for this purpose turns out to be valid provided the hole is much bigger than Planck mass (see e.g. Birrell and Davies [15]). Thus the final fate of an evaporating black hole will not be revealed in this way, though the evolution of the horizon is still of physical interest.

\section{Preliminaries}

Christensen and Fulling [9] showed that the general solution to the conservation equation $\nabla_{v} T_{\mu}^{v}=0$ in Schwarzschild spacetime contains two arbitrary constants and one arbitrary function, which they take to be the component $T_{\theta}^{\theta}$. In addition they assume knowledge of the trace $T_{\alpha}^{\alpha}$, which is given by the trace anomaly. If we require that the energy-momentum tensor be regular on both horizons (past and future) then both the arbitrary constants in the general solution must be zero. This condition applies to the Hartle-Hawking vacuum state. The expressions Christensen and Fulling obtain in this case are:

$$
\begin{aligned}
& T_{r}^{r}=\frac{1}{r^{2}}\left(1-\frac{2 M}{r}\right)^{-1} \int_{2 M}^{r}\left(M T_{\alpha}^{\alpha}\left(r^{\prime}\right)+2\left(r^{\prime}-3 M\right) T_{\theta}^{\theta}\left(r^{\prime}\right)\right) d r^{\prime}, \\
& T_{t}^{t}=T_{\alpha}^{\alpha}-T_{r}^{r}-2 T_{\theta}^{\theta}, \\
& T_{\phi}^{\phi}=T_{\theta}^{\theta} .
\end{aligned}
$$

(I am of course using units in which $G=c=\hbar=1$, and Boltzmann's constant $k$ will also be set to 1.) For a conformally invariant field the trace is given by the anomaly:

$$
T_{\alpha}^{\alpha}=\frac{1}{60 \pi^{2}} \frac{M^{2}}{r^{6}}
$$

The off-diagonal elements of $T_{\mu}^{\nu}$ are zero for a hole in equilibrium. 
Calculations in the Hartle-Hawking state are conveniently performed in the Euclidean section of the Schwarzschild spacetime, obtained by rotating the time coordinate $t$ by $\pi / 2$ in the complex plane. The line element is then:

$$
d s^{2}=\left(1-\frac{2 M}{r}\right) d \tau^{2}+\left(1-\frac{2 M}{r}\right)^{-1} d r^{2}+r^{2} d \theta^{2}+r^{2} \sin ^{2} \theta d \phi^{2},
$$

where $\tau=-i t$.

The singularity in this metric at $r=2 M$ is merely a coordinate singularity if the coordinate $\tau$ is identified with period $8 \pi M$. Thus quantities defined on the Euclidean section of the Schwarzschild spacetime will be periodic in imaginary time. It is a characteristic of thermal Green functions that they are periodic in imaginary time with period $\beta=1 / T$ (see Gibbons and Perry [10], who also show that the Green function defined on the Euclidean section of a static spacetime, with the boundary condition of dying away for large separation of its arguments, is the analytic continuation of the usual Feynman propagator, i.e. it has the correct singularity structure). Thus the thermal nature of the Hartle-Hawking state is expressed through the periodicity in $\tau$, the temperature being given by $T=\frac{1}{\beta}=\frac{1}{8 \pi M}$.

\section{Heat Kernel Formalism and Regularisation}

The Euclidean action for a massless scalar field with arbitrary coupling to the scalar curvature is:

$$
S=\frac{1}{2} \int d^{4} x \sqrt{g}\left(g^{\mu v} \nabla_{\mu} \phi \nabla_{v} \phi+\xi R \phi^{2}\right) .
$$

The conformally invariant theory is obtained by setting $\xi=\frac{1}{6}$. The Green function is the solution of (3.2) which dies away for large separation of the points and is regular on the horizon:

$$
(-\square+\xi R) G\left(x, x^{\prime}\right)=\delta\left(x, x^{\prime}\right)
$$

where $\square \equiv g^{\mu \nu} \nabla_{\mu} \nabla_{v^{*}}$

The energy-momentum tensor is calculated by taking the regularised coincidence limits of the Green function and its derivatives. To isolate the divergences I use the heat kernel method, a Euclidean version of the De Witt-Schwinger proper time formalism. The Green function is expressed as :

$$
G\left(x, x^{\prime}\right)=\int_{0}^{\infty} K\left(x, x^{\prime}, u\right) d u
$$

where the heat kernel $K\left(x, x^{\prime}, u\right)$ is the solution of

$$
\frac{\partial K\left(x, x^{\prime}, u\right)}{\partial u}+\left(-\square_{x}+\xi R(x)\right) K\left(x, x^{\prime}, u\right)=0
$$

with the boundary condition $K\left(x, x^{\prime}, 0\right)=\delta\left(x, x^{\prime}\right)$. Here $K$ will be periodic in imaginary time $\tau$ and will die away for large separations of $x$ and $x^{\prime}$ in spatial 
directions. Also $K$ has a representation in terms of the eigenvalues and eigenfunctions of the operator $-\square+\xi R$ :

$$
K\left(x, x^{\prime}, u\right)=\sum_{\alpha} \phi_{\alpha}(x) \phi_{\alpha}\left(x^{\prime}\right) e^{-\lambda_{\alpha} u},
$$

where

$$
(-\square+\xi R) \phi_{\alpha}=\lambda_{\alpha} \phi_{\alpha}
$$

The sum over $\alpha$ includes an integral over any continuous parameter.

The divergences in coincidence limits of the Green function and its derivatives come from the behaviour of $K\left(x, x^{\prime}, u\right)$ for small values of $u$. This behaviour can be investigated through a representation of $K$ based on the WKB approximation (see e.g. Christensen [16]):

$$
K\left(x, x^{\prime}, u\right)=\frac{\Delta^{\frac{1}{2}}\left(x, x^{\prime}\right)}{16 \pi^{2} u^{2}} \exp \left(-\frac{\sigma\left(x, x^{\prime}\right)}{2 u}\right) \Lambda\left(x, x^{\prime}, u\right),
$$

where $\sigma\left(x, x^{\prime}\right)=\frac{1}{2}$ square of geodetic interval between $x$ and $x^{\prime}$

$$
\Delta\left(x, x^{\prime}\right)=g^{-\frac{1}{2}}(x) \operatorname{det}\left(-\frac{\partial^{2} \sigma\left(x, x^{\prime}\right)}{\partial x^{\mu} \partial x^{v^{\prime}}}\right) g^{-\frac{1}{2}}\left(x^{\prime}\right) .
$$

When $x$ is near $x^{\prime}$ and $u$ is small, $\Lambda$ can be expanded as an asymptotic series in $u$ :

$$
\Lambda\left(x, x^{\prime}, u\right)=\sum_{i=0}^{\infty} a_{i}\left(x, x^{\prime}\right) u^{i}
$$

Christensen [16] gives the coincidence limits of the first few coefficients and their derivatives, found by substituting equation (3.7) into (3.4) and obtaining recurrence relations for the $a_{i}$. For Schwarzschild one finds :

$$
\begin{aligned}
a_{0}(x, x) & =1, \\
a_{1}(x, x) & =0,\left.\quad \nabla_{\mu} a_{\phi}\left(x, x^{\prime}\right)\right|_{x=x^{\prime}}=0, \\
\left.\nabla_{\mu} \nabla_{v} a_{1}\left(x, x^{\prime}\right)\right|_{x=x^{\prime}} & =\frac{1}{90} R^{\varrho \kappa \tau},{ }_{\mu} R_{\varrho \kappa \tau \nu}=\frac{2}{15} g_{\mu \nu} \frac{M^{2}}{r^{2}}, \\
a_{2}(x, x) & =\frac{1}{180} R_{\varrho \kappa \tau i} R^{\varrho \kappa \tau i}=\frac{4}{15} \frac{M^{2}}{r^{6}} .
\end{aligned}
$$

The energy-momentum tensor obtained by varying the action Eq. (3.1) with respect to the metric is:

$$
\begin{aligned}
T_{\mu \nu} \equiv & 2 \frac{1}{\sqrt{g}} \frac{\delta S}{\delta g^{\mu \nu}} \\
= & (1-2 \xi) \nabla_{\mu} \phi \nabla_{\nu} \phi+\left(2 \xi-\frac{1}{2}\right) g_{\mu \nu} \nabla_{\sigma} \phi \Gamma^{\sigma} \phi \\
& -2 \xi \phi \nabla_{\mu} \nabla_{\dot{v}} \phi+2 \xi g_{\mu \nu} \phi \square \phi \\
& +\xi\left(R_{\mu \nu}-\frac{1}{2} g_{\mu \nu} R\right) \phi^{2} .
\end{aligned}
$$


The last term is zero because the background metric is a vacuum solution of Einstein's equations, so I will drop this term. The energy-momentum tensor above is conserved, and if $\xi=\frac{1}{6}$ is traceless, because of the field equations:

$$
\frac{1}{\sqrt{g}} \frac{\delta S}{\delta \phi}=(-\square+\xi R) \phi=0
$$

The expectation value of the operator version of this energy-momentum tensor is given in terms of the heat kernel by:

$$
\begin{aligned}
\left\langle T_{\mu \nu}\right\rangle= & \int_{0}^{\infty} d u\left[\left\{(1-2 \xi) \nabla_{\mu} \nabla_{v^{\prime}}+\left(2 \xi-\frac{1}{2}\right) g_{\mu \nu} \nabla_{\sigma} \nabla^{\sigma^{\prime}}\right.\right. \\
& \left.\left.-2 \xi \nabla_{\mu^{\prime}} \nabla_{v^{\prime}}+2 \xi g_{\mu \nu} \square^{\prime}\right\} K\left(x, x^{\prime}, u\right)\right]_{x=x^{\prime}} \quad \text { regularised }
\end{aligned}
$$

The divergent terms in this integral can be isolated using the representation equation (3.7) and the expansion equation (3.8). In removing the divergences it is necessary to ensure that $\left\langle T_{\mu \nu}\right\rangle$ is conserved. One finds then that the trace of $\left\langle T_{\mu \nu}\right\rangle$ is not zero for a conformally invariant field (i.e. $\xi=\frac{1}{6}$ ). This is the famous trace anomaly.

The resultant conserved, finite energy-momentum tensor is:

$$
\begin{aligned}
\left\langle T_{\mu \nu}\right\rangle= & \int_{0}^{\infty} d u\left[\left\{\nabla_{\mu} \nabla_{v^{\prime}}+\frac{1}{4} g_{\mu v} \square^{\prime}\right\} K\left(x, x^{\prime}, u\right)\right]_{x=x^{\prime}} \\
& +\frac{1}{64 \pi^{2}} g_{\mu \nu} a_{2}(x, x)+\left(\xi-\frac{1}{4}\right) g_{\mu v} \square\left\langle\phi^{2}\right\rangle-\xi \nabla_{\mu} \nabla_{v}\left\langle\phi^{2}\right\rangle,
\end{aligned}
$$

where I have used

$$
\nabla_{\mu} \nabla_{v}\left\langle\phi^{2}\right\rangle=2\left\langle\nabla_{\mu} \phi \nabla_{v} \phi+\phi \nabla_{\mu} \nabla_{v} \phi\right\rangle
$$

Also,

$$
\left\langle\phi^{2}\right\rangle=\int_{0}^{\infty} d u\left[K(x, x, u)-\frac{1}{16 \pi^{2} u^{2}}\right]
$$

is of some physical interest in its own right, in a discussion of spontaneous symmetry breaking near a black hole (Fawcett and Whiting [17]).

Using the representation equation (3.5) of $K$ in terms of the eigenfunctions of the wave operator, we find:

$$
\left\langle\phi^{2}(x)\right\rangle=\int_{0}^{\infty} d u\left[\sum_{\alpha} \phi_{\alpha}^{2}(x) e^{-\lambda \alpha u}-\frac{1}{16 \pi^{2} u^{2}}\right] .
$$

The first term in the expression for $\left\langle T_{\mu \nu}\right\rangle$ is :

$$
\int_{0}^{\infty} d u\left[\sum_{\alpha} \nabla_{\mu} \phi_{\alpha} \nabla_{\nu} \phi_{\alpha}-\frac{1}{4} g_{\mu \nu} \sum_{\alpha} \lambda_{\alpha} \phi_{\alpha}^{2}\right] e^{-\lambda \alpha u} .
$$


Equation (3.13) implies that the trace of $\left\langle T_{\mu \nu}\right\rangle$ is given by

$$
\left\langle T_{\mu}^{\mu}\right\rangle=\left(3 \xi-\frac{1}{2}\right) \square\left\langle\phi^{2}\right\rangle+\frac{1}{16 \pi^{2}} a_{2}
$$

which is $\frac{1}{16 \pi^{2}} a_{2}$ when $\xi=\frac{1}{6}$.

\section{Detailed Expressions for $\left\langle T_{\mu \nu}\right\rangle$}

To evaluate the expressions for $\left\langle T_{\mu \nu}\right\rangle$ it is necessary to find the eigenfunctions of the operator $(-\square+\xi R)$ which in Euclidean Schwarzschild spacetime is $(R=0)$ :

$$
\begin{gathered}
-\left(1-\frac{2 M}{r}\right)^{-1} \frac{\partial^{2}}{\partial \tau^{2}}-\frac{1}{r} \frac{\partial}{\partial r}\left(r(r-2 M) \frac{\partial}{\partial r}\right) \\
-\frac{1}{r^{2}} \frac{1}{\sin \theta} \frac{\partial}{\partial \theta}\left(\sin \theta \frac{\partial}{\partial \theta}\right)-\frac{1}{r^{2} \sin ^{2} \theta} \frac{\partial^{2}}{\partial \phi^{2}}
\end{gathered}
$$

The equation $-\square \phi_{\alpha}=\lambda_{\alpha} \phi_{\alpha}$ is separable:

$$
\phi_{\alpha}=\phi_{n l m p}=T_{n}(\tau) Y_{l m}(\theta, \phi) R_{n l p}(r),
$$

where

$$
T_{n}(\tau)=\frac{1}{\sqrt{\beta}} \exp \left(i \frac{2 \pi}{\beta} n \tau\right), \quad n=0, \pm 1, \pm 2, \ldots
$$

with normalisation

$$
\int_{0}^{\beta}\left|T_{n}\right|^{2} d \tau=1
$$

The $Y_{l m}(\theta, \phi)$ are the usual spherical harmonic normalised so that:

$$
\int_{4 \pi}\left|Y_{l m}(\theta, \phi)\right|^{2} d \Omega=1
$$

The radial functions $R_{n l p}(r)$ satisfy:

$$
\frac{1}{r^{2}} \frac{d}{d r}\left(r(r-2 M) \frac{d R_{n l p}}{d r}\right)+\left(\lambda_{n l p}-\frac{1}{r^{2}} l(l+1)-\left(\frac{2 \pi}{\beta}\right)^{2} n^{2} \frac{r}{r-2 M}\right) R_{n i p}=0 .
$$

The parameter $p$ labels radial eigenvalues - it would be discrete if the black hole were enclosed in a spherical box with some boundary condition imposed, or continuous for a hole in an infinite volume.

Using Eq. (3.5) we obtain for the coincidence limit of the heat kernel:

$$
K(x, x, u)=\frac{1}{4 \pi \beta} \sum_{n=0}^{\infty} d n \sum_{l=0}(2 l+1) \sum_{p} R_{n l p}^{2} \exp \left(-\lambda_{n l p} u\right),
$$

where $d_{0}=1, d_{n}=2$ if $n \geqq 1$ and I have used

$$
\sum_{m=-l}^{l}\left|Y_{l m}\right|^{2}=\frac{1}{4 \pi}(2 l+1) \text {. }
$$


If the black hole is in a spherical box at radius $r_{0}$ then the radial functions should be normalised by:

$$
\int_{2 M}^{r_{0}} R_{n l p}^{2} r^{2} d r=1
$$

We are interested in the case $r_{0}=\infty$, when the parameter $p$ will become continuous and the sum replaced by an integral. To obtain the appropriate expression we need to change the normalisation of the radial functions and, using an asymptotic form of the eigenvalues, take the limit $r_{0} \rightarrow \infty$.

First we remove all explicit dependence of $R$ on $M$ by introducing a new coordinate $x=\frac{r}{2 M}$, and a new eigenvalue $\bar{\lambda}=(2 M)^{2} \lambda$ :

$$
\frac{1}{x^{2}} \frac{d}{d x}\left(x(x-1) \frac{d R_{n l p}}{d x}\right)+\left(\bar{\lambda}-\frac{1}{x^{2}} l(l+1)-\frac{n^{2}}{4} \frac{x^{3}}{x-1}\right) R_{l n p}=0
$$

Using the logarithmic coordinate $x^{*}=x+\ln (x-1)$ and letting $y_{n l p}=x R_{n l p}$, we get:

$$
\frac{d^{2} y_{n l p}}{d x^{* 2}}=\left[\frac{n^{2}}{4}-\left(1-\frac{1}{x}\right)\left(\bar{\lambda}-\frac{1}{x^{2}} l(l+1)-\frac{1}{x^{3}}\right)\right] y_{n l p} .
$$

For large $x^{*}$, the solutions $y_{n l p}$ will oscillate with approximately constant amplitude. We choose this amplitude to be 1 . Then, using the asymptotic form of the eigenvalues :

$$
p \pi \sim\left(\bar{\lambda}-\frac{n^{2}}{4}\right)^{\frac{1}{2}} x^{*}+\mathcal{O}\left(\ln x_{0}^{*}\right)
$$

where $x_{0}^{*}=\frac{r_{0}^{*}}{2 M}, r_{0}^{*}=r_{0}+2 M \ln \left(\frac{r_{0}}{2 M}-1\right)$, it is easy to show that

$$
\operatorname{lt}_{r_{0} \rightarrow \infty} \sum_{p} R_{n l p}^{2} \exp \left(-\lambda_{n l p} u\right)=\frac{1}{x^{2}} \frac{1}{(2 M)^{3}} \frac{1}{\pi} \int_{\frac{n^{2}}{4}}^{\infty} d \bar{\lambda} \frac{y^{2}}{\sqrt{\bar{\lambda}-\frac{n^{2}}{4}}} \exp \left(-\frac{\bar{\lambda}}{(2 M)^{2}} u\right)
$$

The lower limit on the integral arises because there are no normalisable eigenfunctions if $\lambda<\frac{n^{2}}{4}$. The full expression for the coincidence limit of the heat kernel is then:

$$
K(x, x, u)=\frac{16 \pi}{\beta^{4}} \frac{1}{x^{2}} \int_{0}^{\infty} d \bar{\lambda} \sum_{n<2 \sqrt{\bar{\lambda}}} d_{n} \sum_{l=0}^{\infty}(2 l+1) \frac{y_{n l}^{2}}{\sqrt{\bar{\lambda}-\frac{n^{2}}{4}}} \exp \left(-\frac{\bar{\lambda}}{(2 M)^{2}} u\right),
$$

where the order of the sums and integral has been changed. 
We can now write down $\left\langle\phi^{2}\right\rangle$ in terms of the radial functions $y_{n l}$ :

$$
\left\langle\phi^{2}\right\rangle=\frac{1}{\beta^{2}} \int_{0}^{\infty} d \bar{u} \int_{0}^{\infty} d \bar{\lambda}\left[\frac{1}{\pi x^{2}} \sum_{n<2 \sqrt{\bar{\lambda}}} d_{n} \sum_{l}(2 l+1) \frac{y^{2}}{\sqrt{\bar{\lambda}-\frac{n^{2}}{4}}}-\bar{\lambda}\right] e^{-\bar{\lambda} \bar{u}},
$$

where $\bar{u}=\frac{u}{(2 M)^{2}}=\frac{16 \pi^{2}}{\beta^{2}} u$ and I have used $\int_{0}^{\infty} \bar{\lambda} e^{-\bar{\lambda} \bar{u}} d \bar{\lambda}=\frac{1}{\bar{u}^{2}}$.

In a similar way we can obtain an expression for $\left\langle\left(\nabla_{\theta} \phi\right)^{2}+\frac{1}{4} g_{\theta \theta} \phi \square \phi\right\rangle$ required for the $T_{\theta \theta}$ component of the energy-momentum tensor. Because of the $\theta$ derivative we need the result:

$$
\sum_{m=-l}^{l}\left|\frac{\partial Y_{l m}}{\partial \theta}\right|^{2}=\frac{1}{8 \pi}(2 l+1) l(l+1)
$$

We find:

$$
\begin{aligned}
& \left\langle\left(\nabla_{\theta} \phi\right)^{2}+\frac{1}{4} g_{\theta \theta} \phi \square \phi\right\rangle \\
& =\frac{1}{\beta^{2}} \int_{0}^{\infty} d \bar{u} \int_{0}^{\infty} d \bar{\lambda} e^{-\bar{\lambda} \bar{u}}\left[\frac{1}{2 \pi x^{2}} \sum_{n<2 \sqrt{\bar{\lambda}}} d_{n} \sum_{l}(2 l+1) l(l+1) \frac{y^{2}}{\sqrt{\bar{\lambda}-\frac{n^{2}}{4}}}\right. \\
& \left.\quad-\frac{1}{4} x^{2} \lambda \frac{1}{\pi x^{2}} \sum_{n} d_{n} \sum_{l}(2 l+1) \frac{y^{2}}{\sqrt{\bar{\lambda}-\frac{n^{2}}{4}}}\right] .
\end{aligned}
$$

Then $\left\langle T_{\theta \theta}\right\rangle$ is found from Eq. (3.13), and the other components of $\left\langle T_{\mu \nu}\right\rangle$ using the results of Christensen and Fulling, Eq. (2.1).

It is tempting in Eqs. (4.14) and (4.16) to change the orders of the $\bar{\lambda}$ and $\bar{u}$ integrations and perform that over $\bar{u}$ immediately. However, the resultant $\bar{\lambda}$ integrations are not guaranteed to converge, and in fact neither of them does. However, it is permissible to put a lower bound on the $\bar{u}$ integration, change the order of the integrals, and then take the limit as the lower bound tends to zero. For example,

$$
\left\langle\phi^{2}\right\rangle=\operatorname{lt}_{\tilde{u} \rightarrow 0} \frac{1}{\beta^{2}} \int_{0}^{\infty} d \bar{\lambda}\left[\frac{1}{\pi \bar{\lambda}} \frac{1}{x^{2}} \sum_{n} d_{n} \sum_{l}(2 l+1) \frac{y^{2}}{\sqrt{\bar{\lambda}-\frac{n^{2}}{4}}}-1\right] e^{-\bar{\lambda} \bar{u}} .
$$

\section{Numerical Methods}

There are several steps in the numerical evaluation of the expressions derived above. I shall not go into great detail, but will briefly describe the methods used. Programmes were written in the language Algol 68, compiled with a local compiler at Cambridge University, and run on an IBM 370/165 machine.

The first numerical problem is that of finding the normalised eigenfunctions $y(x)$. The numerical integration of Eq. (4.10) was carried out using the Numerov method (Lambert [18]), which is designed for second order ordinary differential 
equations with no first derivative term. It requires two initial values, which were provided by the regular series solution of Eq. (4.9) about the point $x=1\left(x^{*}=-\infty\right)$ corresponding to the horizon. Integration was begun at a large negative value of $x^{*}$ and carried out in $x^{*}$ coordinates to a large positive value. Normalisation was accomplished by matching the numerical solution to a WKB approximate solution at a sufficiently large radius.

One can see from Eq. (4.14) and (4.16) that the integrands in these expressions require two sums to be evaluated. The sum over $n$ is not a problem as it is over a finite range $-n$ is constrained to be less than $2 \sqrt{\bar{\lambda}}$. The sum over $l$, however has no upper limit. It turns out that for given fixed values of $x, n$ and $\bar{\lambda}$ there is a value of $l$ above which contributions to the sum decrease rapidly. This $l$ is given approximately by that value at which the function in square brackets on the right side of Eq. (4.10) changes sign at the given $x$, i.e.

$$
l(l+1)=x^{2}\left[\bar{\lambda}-\frac{1}{x^{3}}-\frac{n^{2}}{4} \frac{x}{x-1}\right] .
$$

Thus the sum over $l$ can be cut off when the contributions become sufficiently small, at some $l$ above the critical value. Because the critical value rises with $x^{2}$ and $\bar{\lambda}$, there are restrictions on how large these quantities can be taken - the computer time needed becomes prohibitive. In fact $I$ was able to calculate $\left\langle T_{\mu \nu}\right\rangle$ out to a radius of only $x^{*}=2$ or $r^{*}=4 M$. The integrand was calculated out to about $\bar{\lambda}=20$.

The integrands in both integrals are oscillating functions of $\sqrt{\bar{\lambda}}$ with periods that depend on $x$, so it is natural to integrate with respect to $\sqrt{\bar{\lambda}}$. Apart from the exponential factor, that for $\left\langle\phi^{2}\right\rangle$ has an envelope that becomes constant at large $\sqrt{\bar{\lambda}}$, while the envelope of the integrand in the expression for $\left\langle\left(\nabla_{\theta} \phi\right)^{2}+\frac{1}{4} g_{\theta \theta} \phi \square \phi\right\rangle$ grows as $\bar{\lambda}$ for large $\sqrt{\bar{\lambda}}$. Thus if $\bar{u}$ is set to zero, neither integral converges.

For the integral needed for $\left\langle\left(\nabla_{\theta} \phi\right)^{2}+\frac{1}{4} g_{\theta \theta} \phi \square \phi\right\rangle$ an additional integration with respect to $\bar{u}$ was performed first, so that the envelope of the oscillating integrand

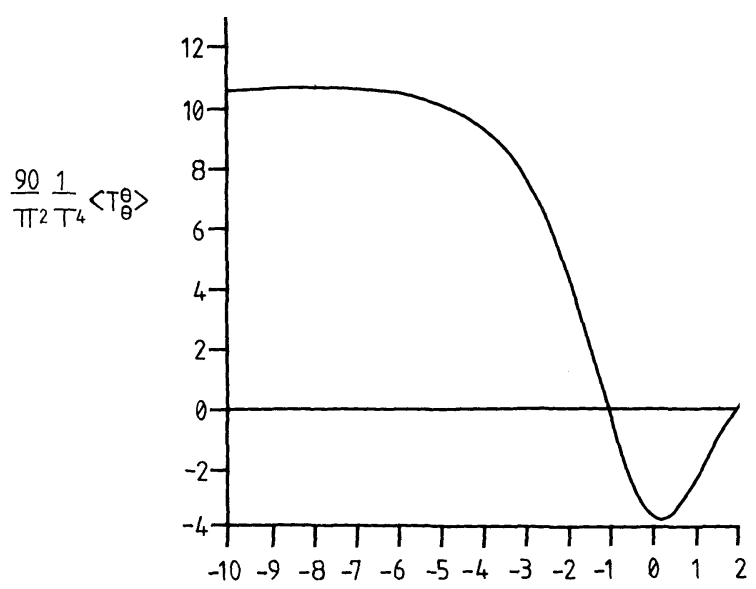

Fig. 1. $\left\langle T_{\phi}^{\phi}\right\rangle$ for a massless conformally invariant field 


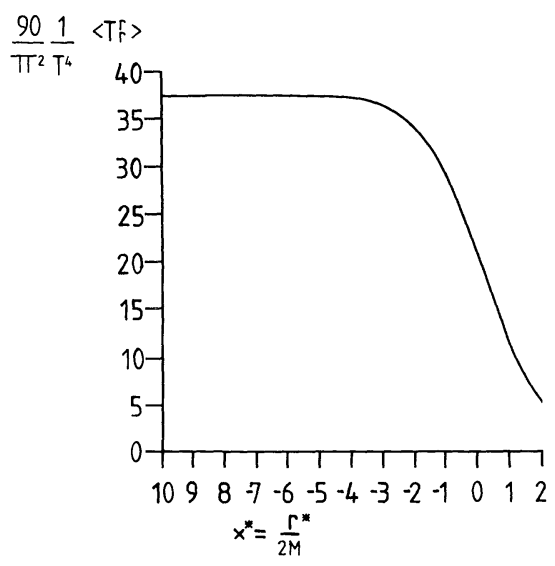

Fig. 2. $\left\langle T_{r}^{r}\right\rangle$ for a massless conformally invariant field

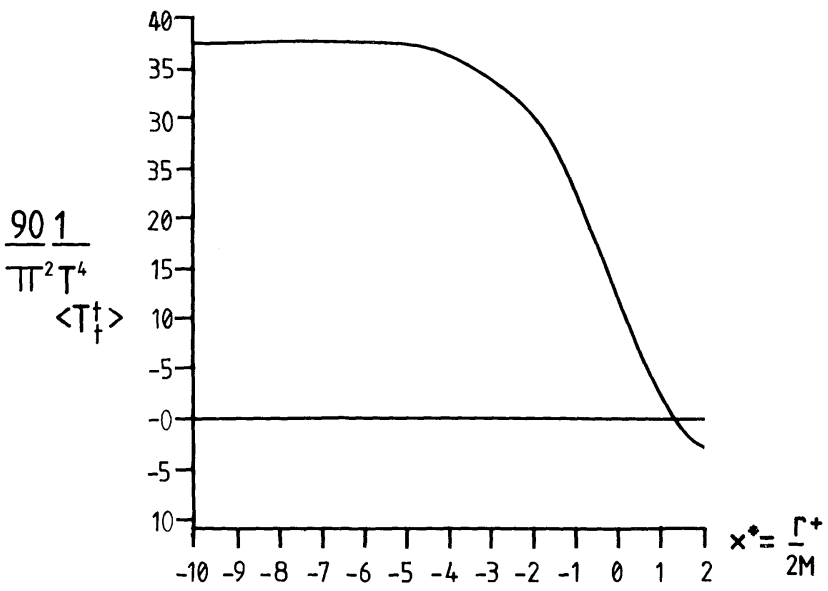

Fig. 3. $\left\langle T_{t}^{t}\right\rangle$ for a massless conformally invariant field

became constant for large $\sqrt{\bar{\lambda}}$. Then after the integration with respect to $\sqrt{\bar{\lambda}}$, a derivative with respect to $\bar{u}$ was taken.

The integrals are best done numerically by integrating over periods to obtain an oscillating sum. This can then be subjected to an Euler transformation (see e.g. Ralston and Rabinowitz [19]) which is designed to speed the convergence of oscillating sums. One finds that the transformed sums converge even when $\bar{u}=0$, hence the limit may be taken immediately. The convergence is rapid, so knowing the integrand only for $\bar{\lambda}<20$ gives sufficient accuracy.

\section{Results}

The components of the energy-momentum tensor for a conformally invariant field are shown in Figs. 1-3. The components have the same values in the Euclidean 


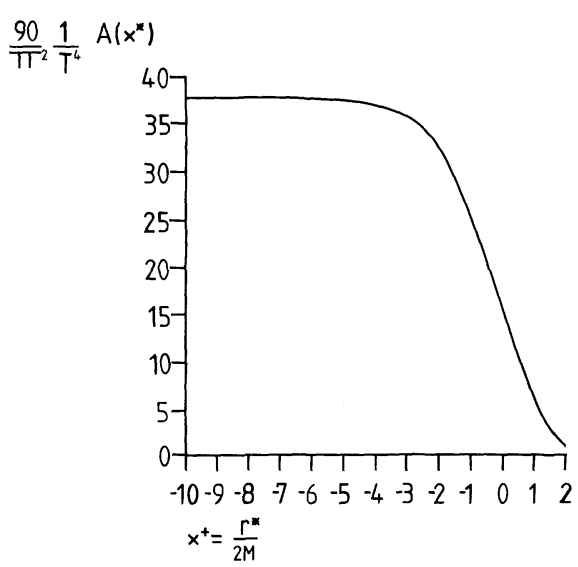

Fig. 4. $A(r)=\frac{1}{2}\left(\left\langle T_{t}^{t}\right\rangle+\left\langle T_{r}^{r}\right\rangle\right)$

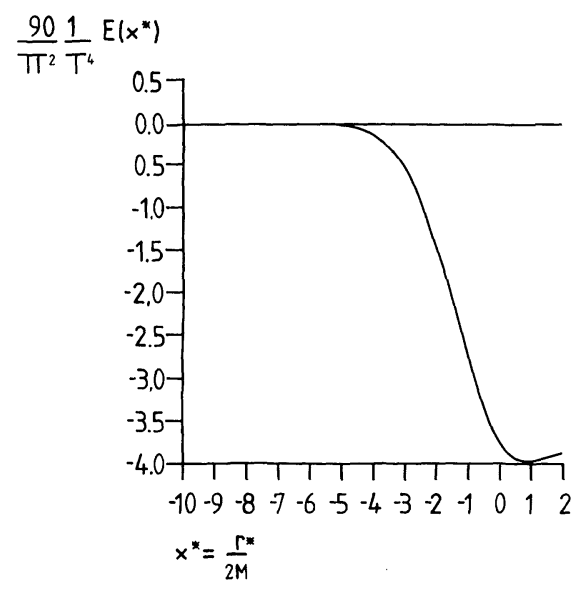

Fig. 5. $E(r)=\frac{1}{2}\left(\left\langle T_{t}^{t}\right\rangle-\left\langle T_{r}^{r}\right\rangle\right)$

and Lorentzian sections, but the coordinate $t$ is pathological at $r=2 M$ in the physical metric. In the well-behaved Kruskal coordinate system,

$$
\begin{aligned}
& u=-\left(\frac{r}{2 M}-1\right)^{1 / 2} e^{(r-t) / 4 M} \\
& v=\left(\frac{r}{2 M}-1\right)^{1 / 2} e^{(r+t) / 4 M}
\end{aligned}
$$

the components are:

$$
\begin{aligned}
& T_{u}^{u}=T_{v}^{v}=\frac{1}{2}\left(T_{t}^{t}+T_{r}^{r}\right), \\
& T_{v}^{u}=\frac{r}{2 M} e^{-t / 2 M} \frac{1}{2}\left(T_{t}^{t}-T_{r}^{r}\right), \\
& T_{u}^{v}=\frac{r}{2 M} e^{t / 2 M} \frac{1}{2}\left(T_{t}^{t}-T_{r}^{r}\right) .
\end{aligned}
$$

The functions

$$
A(r)=\frac{1}{2}\left(T_{t}^{t}+T_{r}^{r}\right) \quad \text { and } \quad E(r)=\frac{1}{2}\left(T_{t}^{t}-T_{r}^{r}\right)
$$

are shown in Figs. 4 and 5. (The notation is chosen to be consistent with Candelas [1] and Page [2].)

The component $\left\langle T_{t}^{t}\right\rangle$ gives the negative of the energy density, $\varrho=-\left\langle T_{t}^{t}\right\rangle$. Thus the energy density is negative near the horizon, out to a radius of about $r=3.5 \mathrm{M}$, after which it is positive. A negative energy density near the horizon is not surprising as it is necessary to violate the weak energy condition in order to get around Hawking's theorem that the area of a black hole can only increase (Hawking and Ellis [20]). We obviously expect the area of the horizon to go down as a hole radiates. 
The transverse pressures $\left\langle T_{\theta}^{\theta}\right\rangle$ and $\left\langle T_{\phi}^{\phi}\right\rangle$ are also negative over a range of radii from about $2.25 \mathrm{M}$ to $4 \mathrm{M}$, while the radial pressure $\left\langle T_{r}^{r}\right\rangle$ is always positive.

Candelas [1] has done some calculations with which my results may be compared. He calculated $\left\langle\phi^{2}\right\rangle$ exactly on the horizon, obtaining a value of $\frac{T^{2}}{3}$. My numerical result agrees with this to about one part in $10^{4}$. He also calculates (effectively)

and

$$
T_{\theta}^{\theta}(2 M)=10.29 \frac{\pi^{2}}{90} T^{4}
$$

$$
A(2 M)=37.71 \frac{\pi^{2}}{90} T^{4},
$$

which are to be compared with my results:

$$
\begin{aligned}
T_{\theta}^{\theta}(2 M) & =10.49 \frac{\pi^{2}}{90} T^{4} \\
A(2 M) & =37.53 \frac{\pi^{2}}{90} T^{4} .
\end{aligned}
$$

The differences are within my expected errors.

Calculations have also been done by Page [2] using the Gaussian approximation of Bekenstein and Parker [21] to the path integral formula for the Green function. Page obtains a result for $\left\langle\phi^{2}\right\rangle$ that is identical to a conjecture of Whiting's (Fawcett and Whiting [17]), and is in excellent agreement with my numerical results for all radii. The greatest difference occurs near $r=3 \mathrm{M}$, where one might expect a contribution to the propagator from indirect geodesics - Page's approximation includes only direct geodesics.

It is perhaps surprising that there are dramatic differences between Page's results and mine for $\left\langle T_{\mu \nu}\right\rangle$. Page's $\varrho=-\left\langle T_{t}^{t}\right\rangle$ has a maximum value nearer the horizon, but is otherwise not too different from mine. The radial pressure $\left\langle T_{r}^{r}\right\rangle$ is very similar to the numerical result. However, the transverse pressure $\left\langle T_{\theta}^{\theta}\right\rangle=\left\langle T_{\phi}^{\phi}\right\rangle$ is dramatically different. The Gaussian approximation quantity is always positive and shows no hint of the dip in the numerical result. It is assumed again that the difference is due to geodesics close to $r=3 M$, though attempts to calculate the effects of these have not been very successful.

\section{Further Calculations}

The most obvious application of a knowledge of $\left\langle T_{\mu \nu}\right\rangle$ is in an investigation of the evolution of the horizon of a black hole as it evaporates. This requires $\left\langle T_{\mu \nu}\right\rangle$ in the Unruh vacuum, i.e. for a black hole radiating into empty space. To obtain this from the Hartle-Hawking $\left\langle T_{\mu \nu}\right\rangle$ it is necessary to subtract the contribution from incoming thermal radiation. This calculation has not yet been done, though it presents no great difficulties in principle. The evolution of the horizon would then also need to be investigated numerically. It is hoped that these calculations will be done in the not too distant future. 
Acknowledgements. I would like to thank Professor S. W. Hawking for suggesting this topic of research, and Dr. B. Whiting and Professor D. Page for many helpful discussions.

\section{References}

1. Candelas, P.: Vacuum polarization in Schwarzschild spacetime. Phys. Rev. D21, 2185-2202 (1980)

2. Page, D.N.: Thermal stress tensors in static Einstein spaces. Phys. Rev. D 25, 1499-1509 (1982)

3. Hawking, S.W.: Particle creation by black holes. Commun. Math. Phys. 43, 199-220 (1975)

4. Hartle, J.B., Hawking, S.W.: Path integral derivation of black hole radiance. Phys. Rev. D13, 2188-2203 (1976)

5. Boulware, D.B.: Quantum field theory in Schwarzschild and Rindler spaces. Phys. Rev. D11, 1404-1423 (1975)

6. Unruh, W.G. : Notes on black hole evaporation. Phys. Rev: D14, 870-892 (1976)

7. Wald, R.M.: On particle creation by black holes. Commun. Math. Phys. 45, 9-34 (1975)

8. Davies, P.C.W., Fulling, S.A., Unruh, W.G. : Energy momentum tensor near an evaporating black hole. Phys. Rev. D13, 2720-2723 (1976)

9. Christensen, S.M., Fulling, S.A.: Trace anomalies and the Hawking effect. Phys. Rev. D15, 2088-2104 (1977)

10. Gibbons, G.W., Perry, M.J.: Black holes and thermal Green's functions. Proc. R. Soc. A358, 467-494 (1978)

11. Gibbons, G.W.: Quantum field theory in curved spacetime. In: General relativity. Hawking, S.W., Israel, W. (eds.). Cambridge: Cambridge University Press 1979

12. Gibbons, G.W.: Quantization about classical background metrics. Talk given at the 9 th International Conference on General Relativity and Gravitation, Jena (1980)

13. De Witt, B. : Quantum field theory in curved spacetime. Phys. Rep. 19C, 295-357 (1975)

14. De Witt, B.: Quantum gravity: the new synthesis. In: General relativity. Hawking, S.W., Israel, W. (eds.). Cambridge: Cambridge University Press 1979

15. Birrell, N.D., Davies, P.C.W.: Quantum fields in curved space. Cambridge: Cambridge University Press 1982

16. Christensen, S.M.: Vacuum expectation value of the stress tensor in an arbitrary curved background: the covariant point-separation method. Phys. Rev. D 14, 2490-2501 (1976)

17. Fawcett, M.S., Whiting, B.: Spontaneous symmetry breaking near a black hole. Talk given at Nuffield quantum gravity conference at Imperial College, London (1981)

18. Lambert, J.D.: Computational methods in ordinary differential equations. New York: Wiley 1973

19. Ralston, A., Rabinowitz, P.: A first course in numerical analysis. Tokyo: Kogakusha: McGrawHill 1978

20. Hawking, S.W., Ellis, G.F.R.: The large-scale structure of space-time. Cambridge: Cambridge University Press 1973

21. Bekenstein, J.D., Parker, L. : Path-integral evaluation of Feynman propagator in curved spacetime. Phys. Rev. D23, 2850-2869 (1981)

Communicated by S. Hawking

Received October 29, 1982 
